\title{
Vermal infarct with pursuit eye movement disorders
}

\author{
Charles Pierrot-Deseilligny, Pierre Amarenco, Etienne Roullet, René Marteau
}

\begin{abstract}
Severe deficits of foveal smooth pursuit and optokinetic nystagmus in all directions were electro-oculographically recorded in an 80 year old woman. Magnetic resonance imaging (MRI) showed an infarct involving the postero-inferior part of the vermis (lobules VI to $X$ ) and a portion of the left cerebellar hemisphere, with apparent preservation of the flocculus and the brainstem. The role of the vermal lesion in these pursuit eye movement disorders is discussed.
\end{abstract}

It has recently been shown that, besides the flocculus, the posterior part of the vermis (especially lobules VI and VII) is involved in the control of pursuit eye movements in the monkey..$^{2}$ Our case reports for the first time a recent ischaemic lesion of the posterior vermis and eye movement recordings, suggesting that this cerebellar structure is also involved in the control of pursuit eye movements in humans.

\section{Case report}

An 80 year old woman experienced sudden vertigo, with unsteadiness, vomiting, posterior headache, without auditory symptoms. For the previous five years she had received treatment for high blood pressure. On admission her blood pressure was $160 / 80 \mathrm{~mm} \mathrm{Hg}$, her pulse rate was irregular at 115 per minute. There was no drowsiness. Vertigo increased on change of head posture. Finger-to-nose and rapid alternating movements of the left upper limb were ataxic. Both eyes and head were tonically deviated leftwards. However, oculocephalic movement allowed both eyes to overcome this tonic deviation and reach the extreme right position. Cranial nerves were unaffected. There were no sensory or motor deficits, all tendon reflexes were normal and plantar responses flexor. The electrocardiogram showed atrial fibrillation. CT scan performed on the day of admission was normal. In particular, there was no hydrocephalus, which suggested that there was no compression of the brainstem by the cerebellum.

MRI performed four days later showed an infarct in the territory of the left posterior and inferior cerebellar artery, mainly the territory of its medial branch. ${ }^{3}$ All the inferior part of the vermis was affected (lobules VI to $\mathrm{X}$ ), namely the clivus, the folium, the tuber, the pyramis, the uvula and the nodulus (fig 1). The inferior part of the left cerebellar hemisphere was also damaged. The flocculus, the different cerebellar peduncles and the brainstem were apparently spared. Moreover, the brainstem did not appear to be compressed. There was only slight diffuse atrophy in the cerebral hemispheres, without hydrocephalus. The posterior headache lasted one day, vertigo and left lateropulsion on walking cleared up within several days and the left tonic ocular deviation progressively disappeared within ten days.

\section{Oculographic study}

Eye movements were recorded on the eleventh day after the onset of the symptoms, while the patient was fully alert, cooperative and attentive. She was not taking any other medication than acetyl-salycylic acid. Directcurrent electro-oculography (EOG) was used, for each eye separately, in darkness, with the head immobilised. There was no nystagmus when the patient looked straight ahead or in the extreme positions. With eyes open, there was no tonic deviation, but with eyes closed there was a left conjugate eye deviation.

Horizontal eye movements Saccades to command and visually guided saccades had normal latency, velocity and amplitude (fig $2 \mathrm{~A}$ ). In particular, no dysmetria was observed, whatever the position of the visual target (at $5^{\circ}, 15^{\circ}, 25^{\circ}$ and $35^{\circ}$ away from the midline) and direction of saccades (left, right, or returns to the midline).

Foveal pursuit of a small luminous target moving sinusoidally at peak-to-peak amplitude of $50^{\circ}$ was largely saccadic in both directions (fig 2B). Both catch-up saccades and smooth portions were larger leftwards than rightwards but smooth eye movement velocities were similar in both directions. Smooth movement gains (ratio of peak eye velocity to peak stimulus velocity) were low, bilaterally, compared to an age-matched control group, for the two frequencies of stimulation used (table). These gains were less than the lower limit of the normal range (control mean-2 SD).

Optokinetic nystagmus (OKN) was elicited using alternating black and white stripes moving at $30^{\circ} / \mathrm{s}$ across a screen covering $60^{\circ}$ of the visual field. It was severely impaired (fig $2 \mathrm{C}$ ), 
Figure 1 Magnetic resonance imaging ( $T 2$ ). $A$ and $B$ : horizontal sections passing through the mid-pons and the upper medulla,

respectively. C: coronal section passing through the posterior part of the cerebellum. Note the infarct in the territory of the left posterior and inferior cerebellar artery involving the vermis (lobules VI to X) and the postero-inferior part of the left cerebellar hemisphere. The flocculus (B, arrow), the cerebellar peduncles and the brainstem $(A, B)$ did not appear to be damaged.

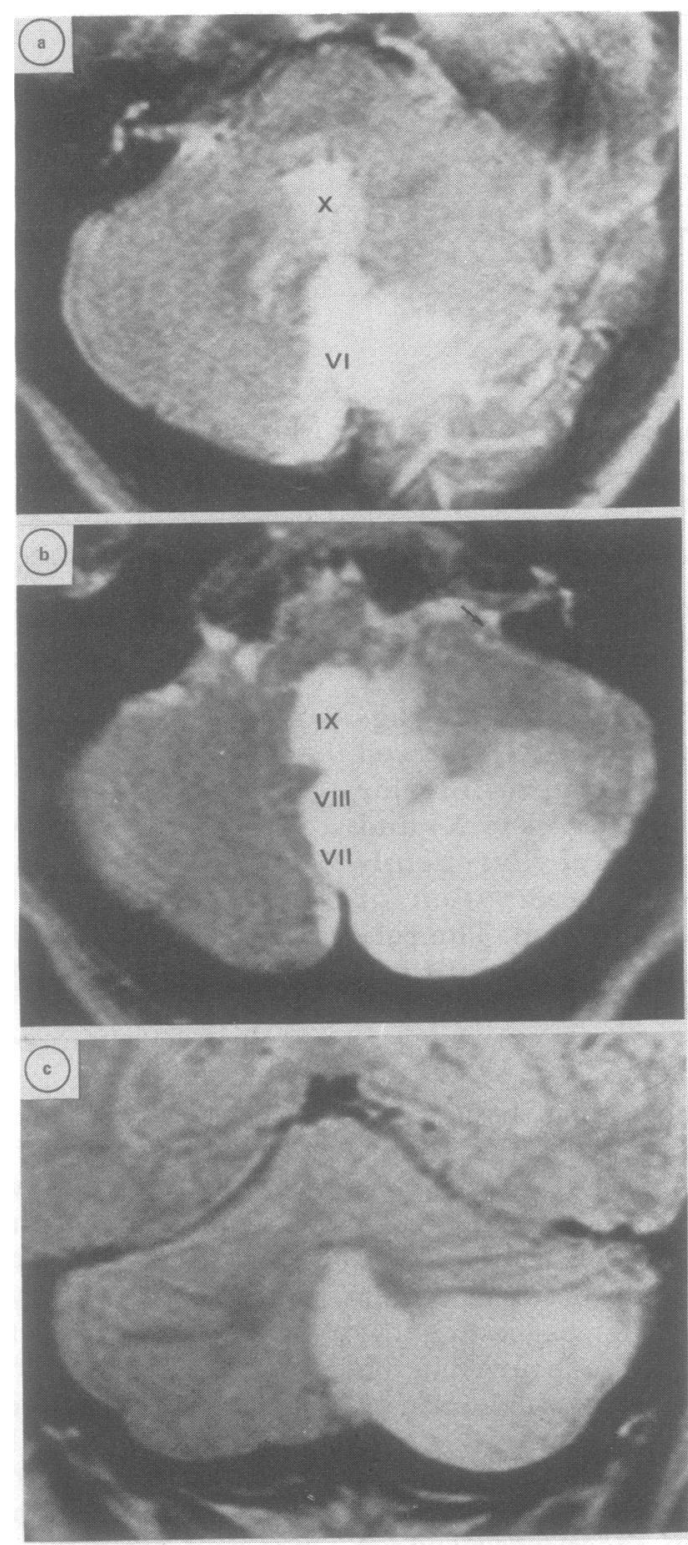

without any build up after sustained stimulation (1 minute). Slow phase gains were very low, bilaterally, compared to the control group (table). These gains were also less than the lower limit of the normal range.

Vestibulo-ocular reflex (VOR) was tested by pseudo-sinusoidal rotation of the chair at peak-to-peak amplitude of $45^{\circ}$ while the patient looked at a small stationary light. The movement elicited, a combination of VOR and smooth pursuit, appeared to be qualitatively normal, with a gain near 1 (fig 2D). Convergence, tested in the light, was weak for both eyes.

Vertical eye movements These movements were not quantified because EOG is unreliable in this plane. Nevertheless, abnormalities of upward and downward eye movement were similar. Saccades appeared to be normal, foveal smooth pursuit was largely saccadic, OKN was absent (without any slow movement), oculocephalic movements (passive rotations of the head) were present, with a full amplitude.

\section{Discussion}

This case included vestibular symptoms, a leftward tonic deviation of the eyes and abnormalities of pursuit eye movements, which will be discussed successively. For several days, there was marked vertigo and leftward axial lateropulsion. Isolated and true vestibular symptoms may be observed in pure cerebellar infarcts, resulting from occlusions of the posterior and inferior cerebellar artery, ${ }^{4}$ or only its medial branch ${ }^{5}$ or of the anterior and inferior cerebellar artery. ${ }^{6}$ The vestibular symptoms could be due to lesions of the flocculus ${ }^{4}$ or of the nodulus and uvula. ${ }^{5}$ As the flocculus was apparently spared here, the vestibular symptoms probably resulted from damage to the nodulus and uvula. This vermal part of the vestibulo-cerebellum is reciprocally connected with the vestibular nuclei. ${ }^{7}$ Thus the impairment of this supravestibular control system

Figure 2 Lateral eye movements (left eye). A visually guided saccades $a$, stimulation; $b$, eye movement, normal. $B$ Foveal pursuit: $a$ stimulation $(0 \cdot 26 \mathrm{~Hz}) ; b$, eye movement, saccadic in both directions. $C$ ) $O K N$ (rightward stimulation and, after the vertical arrow, leftward stimulation): impaired in both directions. D) VOR $a$, stimulation; $b$, eye movement, apparently normal. our patient.

A leftward tonic conjugate eye deviation also existed initially, showing progressive improvement within 10 days and leaving only a leftward deviation during eyelid closure on the eleventh day (when eye movements were recorded). Conjugate tonic deviation of the eyes has previously been reported in haemorrhagic or ischaemic ${ }^{8}$ lesions of the cerebellum. However, those cases included brainstem compression.
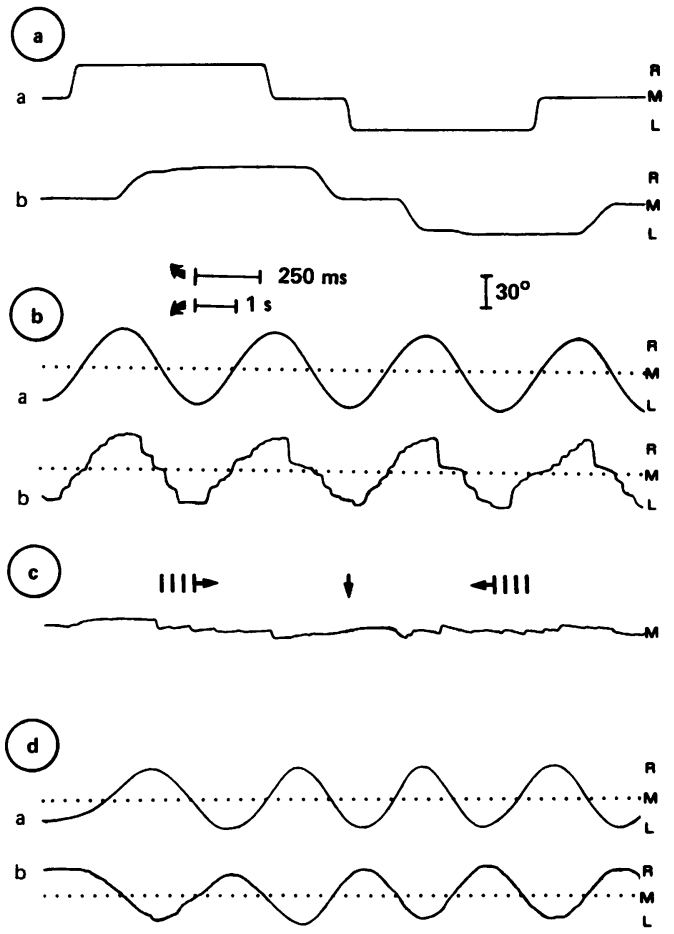
In our patient, MRI showed that the brainstem was apparently spared. Moreover, the absence of drowsiness and the normal appearance of the ventricles (without hydrocephalus) suggested that the brainstem was not compressed by the vermal infarct. Therefore it may be assumed that the tonic ocular deviation was due to the cerebellar lesion. However, it should be noted that in experimental unilateral lesions of the cerebellum $^{9}$ and in a unilateral infarct of the rostral cerebellum ${ }^{10}$ tonic ocular deviation was contralateral to the lesion, whereas it was ipsilateral here. As in our patient, the lesion 
Table Horizontal smooth pursuit gains

\begin{tabular}{lllll}
\hline & & Right gain & Left gain & Normal gain $^{\star}$ \\
\hline $\begin{array}{l}\text { Foveal } \\
\text { smooth pursuit }\end{array}$ & $\begin{array}{l}0.11 \mathrm{~Hz} \text { (peak velocity: } \\
\left.15^{\circ} / \mathrm{s}\right)\end{array}$ & 0.47 & 0.44 & $0.72(0.07)$ \\
& $\begin{array}{l}0.26 \mathrm{~Hz} \text { (peak velocity: } \\
\text { OKN }\end{array}$ & $\left.0.31^{\circ} / \mathrm{s}\right)$ & 0.36 & $0.67(0.09)$ \\
& $\left(30^{\circ} / \mathrm{s}\right)$ & 0.17 & 0.19 & $0.62(0.14)$ \\
\hline
\end{tabular}

* $[$ mean $(S D) ; n=10$, mean age $=76$ years, range $=70-84$ years $]$.

involved all the postero-inferior portion of the vermis along with the inferior part of the left cerebellar hemisphere, but it is difficult to suggest a detailed explanation that would account for this deviation.

Foveal smooth pursuit and OKN were severely imparied in all directions. Our patient was 80 years old and there is usually a slight impairment of pursuit eye movement in the elderly. ${ }^{11}$ However, as the pursuit deficits seen here were much more severe than those of an age-matched control group, it may be considered that old age was not the only cause of these deficits. Drowsiness, inattention and certain medications may also impair pursuit eye movements, but none of these was present. Lastly, as already emphasised, there was no clinical or radiological evidence of damage to, or compression of, the brainstem which could account for this impairment of pursuit eye movements. Therefore, it may be assumed that the cerebellar infarct itself was the primary cause of the eye movement disorders.

It has been shown that the cerebellum is an essential relay in smooth pursuit eye movement circuitry. These movements no longer existed after total cerebellectomy in the monkey. ${ }^{9}$ In the cerebellum, the flocculus is important since the smooth pursuit eye movement gain is nearly $60 \%$ after flocculectomy. ${ }^{12}$ The difference between a simple flocculectomy and a total cerebellectomy suggests that other parts of the cerebellum are involved in smooth pursuit eye movements. In the monkey, the posterior part of the vermis (lobules VI and VII) contains cells involved in these movements ${ }^{2}$ and lesions at this level decrease the

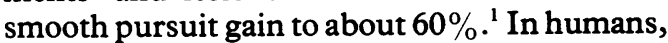
diffuse degenerative lesions of the cerebellum also result in decreased smooth pursuit gain. ${ }^{13}$ Focal lesions with eye movement recordings are rare and our case could be the first to suggest that pursuit eye movement disorders in humans may result from damage to the posterior vermis. Indeed, this region was impaired, while the flocculus and the brainstem seemed to be unaffected. The nodulus and uvula were also damaged in our patient, but these structures do not appear to be involved in pursuit eye movements. ${ }^{14}$ The lesion in the inferior part of the left cerebellar hemisphere might have played a role in the smooth pursuit disorders in our patient. So far, it has not been suggested that such a region could be involved in these movements either experimentally or clinically.

Lastly, saccade accuracy was preserved in our patient. Lesions of the posterior vermis are known to result in saccade dysmetria, in the monkey (in particular when lobules V, VI and VII are damaged) $)^{115}$ as well as in humans. ${ }^{16}$ It is possible that this part of the vermis is in fact less crucial for saccade accuracy, or that adaptative mechanisms were already operative in our patient when eye movements were recorded.

Thus this case suggests that the posterior vermis is, like the flocculus, involved in pursuit eye movements. The activities of the flocculus and posterior vermis cells are not exactly identical during slow eye movements. ${ }^{1}$ In particular, the discharge of vermal cells is proportional to target velocity while that of floccular cells depend on eye and/or head velocity. The projections of the flocculus and the vermis are also noticeably different. ${ }^{1718}$ The former projects on to the medial vestibular nucleus whereas the latter projects (via the fastigial nuclei) on to neurons located near the abducens nucleus and probably involved in smooth pursuit. ${ }^{1}$ These differences in the activity, as well as in the efferent pathways, of the flocculus and vermis are not yet well understood. Further studies are needed to determine the specific roles of these two structures in the control of pursuit eye movements.

We thank Miss S Rivaud for technical assistance.

1 Keller EL. Cerebellar involvement in smooth pursuit eye movement generation. In: Clifford Rose F, Kennard C, eds. Physiological aspects of clinical neuro-ophthalmology. Lods. Physiological aspects of clinical neuro-

2 Suzuki DA, Keller EL. The role of the posterior vermis of monkey cerebellum in smooth-pursuit eye movement monkey cerebellum in smooth-purs

3 Amarenco $\mathrm{P}$, Hauw JJ. Anatomie des artères cèrébelleuses. Rev Neurol 1989;145:267-76.

4 Duncan GW, Parker SW, Fisher CM. Acute cerebellar infarction in the PICA territory. Arch Neurol 1975; 32:364-8.

5 Amarenco P, Hauw JJ, Hénin D, et al. Les infarctus du territoire de l'artère cérébelleuse postérieure et inférieure. Etude clinico-pathologique de 28 cas. Rev Neurol 1989;145:277-86

6 Rubenstein RL, Norman D, Schindler R, Kassef L. Cerebellar infarction: a presentation of vertigo. Laryngoscope 1980;90:505-14.

7 Carleton SC, Carpenter MB. Afferent and efferent connections of the medial, inferior and lateral vestibular nuclei in the cat and monkey. Brain Res 1983;278:29-51.

8 Lehrich JR, Winckler GF, Ojemann RG. Cerebellar infarction with brain stem compression. Arch Neurol 1970;22:490-8.

9 Westheimer G, Blair SM. Functional organization of primate oculomotor system revealed by cerebellectomy. Exp Brain Res 1974;21:463-72.

10 Ranalli PJ, Sharpe JA. Contrapulsion of saccades and ipsilateral ataxia: a unilateral disorder of the rostral cerebellum. Ann Neurol 1986;20:311-6.

11 Büttner U. Smooth pursuit eye movements, optokinetic nystagmus and vestibulo-ocular reflex suppression. In: Clifford Rose F, Kennard C, eds. Physiological aspects of Clinical neuro-ophthalmology. London: Chapman and Hall, 1988:278-92.

12 Zee DS, Yamazaki A, Butler PH, Gücer G. Effects of ablation of flocculus and paraflocculus on eye movements ablation of flocculus and paraflocculus on eye

13 Baloh RW, Yee RD. Honrubia V. Late cortical cerebellar atrophy. Clinical and oculographic features. Brain 1986;109:159-80.

14 Waespe W, Cohen B, Raphan T. Dynamic modification of the vestibulo-ocular reflex by the nodulus and uvula. Science 1985;228:199-202.

15 Ritchie L. Effects of cerebellar lesions on saccadic eye movements. J Neurophysiol 1976;39:1246-56.

6 Selhorst JB, Stark L, Ochs AL, Hoyt WF. Disorders in cerebellar ocular motor control. I. Saccadic overshoo dysmetria: an oculographic, control system and clinicoanatomical analysis. Brain 1976;99:497-508.

17 Langer T, Fuchs AF, Chubb MC, et al. Floccular efferents in the rhesus macaque as revealed by autoradiography and in the rhesus macaque as revealed by autoradiography and

18 Yamada J, Noda $H$. Afferent and efferent connections of the oculomotor cerebellar vermis in the macaque monkey. $J$ Comp Neurol 1987;265:224-41 